TECHNICAL NOTE
A. Chang
S. Pochert
C. Romano
A. Brook
T. Miller

\section{Safety of 1000 CT-Guided Steroid Injections with Air Used to Localize the Epidural Space}

SUMMARY: Historically, ESIs were performed without any imaging guidance, resulting in erroneous placement in up to $30 \%$ of injections. Fluoroscopic imaging is now used to guide most procedures. Recently, several reports have described the use of CT to guide ESIs instead of fluoroscopy. CT provides the ability to use air as contrast to localize the epidural space. This retrospective review will discuss findings in 1000 CT-guided ESIs with air localization.

ABBREVIATIONS: $A D R=$ adverse drug reaction; $E S I=$ epidural spinal injection
E SIs have a low complication rate ${ }^{1}$ and are minimally invasive. While the efficacy of ESIs is still unclear, observational studies have largely supported their effectiveness. However, there are few well-designed randomized double-blinded studies. $^{2}$

Historically, ESIs were performed without any imaging guidance, resulting in erroneous placement in up to $30 \%$ of injections. ${ }^{3}$ Because of this and the potential for intrathecal and intravascular injections, imaging is now used to guide most procedures. Although fluoroscopy is currently the standard for ESIs, it has its limitations. Among these is the poor demonstration of soft-tissue structures, which necessitates contrast injection to localize the epidural space or nerve roots. It has been reported that ESIs performed without contrast are at increased risk of being intravascular injections (transforaminal), ${ }^{4}$ which can, in turn, cause untoward side effects. Furthermore, contrast use runs the risk of inducing allergic reactions in contrast-sensitive patients.

More recently, several reports have described the use of CT to guide ESIs instead of fluoroscopy. CT has several advantages, including increased anatomic precision and visualization of needle placement with millimeter accuracy. The superior tissue contrast offered by CT provides the ability to use air as contrast to localize the epidural space. Air is used in much the same way as iodinated contrast - it is injected to verify that the needle is indeed within the epidural space and not intrathecal. Air has several advantages, including lack of risk of allergic reactions and no cost for materials. There is profound ability to discriminate between air and soft-tissue by using CT, which makes air an excellent contrast agent. Because incorrect needle placement and incorrect steroid injection are the most frequent complications of ESIs, it is important to find a safe and reliable way to localize the epidural space.

Because this method, to our knowledge, has not been previously described in the literature, this retrospective review will discuss findings in 1000 CT-guided ESIs with air localization.

Received June 29, 2010; accepted after revision August 9.

From the Department of Radiology, Montefiore Medical Center, Albert Einstein College of Medicine, Bronx, New York.

Please address correspondence to Todd Miller, MD, Albert Einstein College of Medicine, Montefiore Medical Center Department of Radiology, 111 East 210th St, Bronx, New York, 10467; e-mail: tmiller@montefiore.org

http://dx.doi.org/10.3174/ajnr.A2316

\section{Materials and Methods}

This institutional review board-approved protocol is a retrospective review composed of a record search in the computerized radiology information system from 2006 to 2009. The search included all CTguided ESIs in the lumbar region for which air was used for localization. Cases in which the additional use of contrast was used for needle localization were included as well. The data collected included age, sex, level treated, needle size, amount of air, type and amount of steroid used, contrast use and amount, and any reported complications.

\section{Technique}

The CT-guided epidural access was typically performed with the patient prone; adjustments were made for patient comfort. A standard sterile prep and drape were used. A scout lateral image was obtained. Five axial images through the affected level (3-mm helical, $120 \mathrm{kVp}$, $50 \mathrm{~mA}$ ) were obtained for planning. The trajectory was planned on the CT console. The skin entry site and depth were recorded. Local anesthesia was administered with $2 \%$ lidocaine both superficially and deep at the planned entry location. The needle was inserted $1 \mathrm{~cm}$ short of the planned depth. A 3-mL luer-slip syringe with $1 \mathrm{~mL}$ of air was attached to the spinal needle, and the loss of resistance technique was used to cross the ligamentum flavum. Confirmatory images were obtained once loss of resistance was felt by the operator.

\section{Results}

We identified 751 patients who underwent 1000 procedures. The average patient age was 59.2 years, 439 (58.5\%) were women and $312(41.5 \%)$ were men. Seventy-five percent of the treated levels were L5/S1, with L4/5 and L3/4 much less frequently treated $(15.5 \%$ and $4.9 \%)$. Rarely, procedures targeted L2/3 (1.3\%), L1/2 (0.8\%), and T12/L1 (0.1\%). A spinal needle was used for all procedures, with $90 \%$ using a 22 -ga spinal needle. Of the total number of cases, 575 reported a specific amount of air injected; the typical dose was $0.5 \mathrm{~mL}$, with a range of $0.05-2 \mathrm{~mL}$ (Fig 1 ). A total of 132 cases required further administration of iodinated contrast, predominantly iohexal (Omnipaque 180; GE Healthcare, Princeton, New Jersey), to confirm needle position. Of those cases, 103 reported a specific amount of contrast; the usual dose was $1.0 \mathrm{~mL}$, with a range of $0.5-5.0 \mathrm{~mL}$

The most common steroid administered was betamethasone acetate (Celestone; Schering, Kenilworth, New Jersey) (in $91.5 \%$ of cases), with methylprednisolone acetate (DepoMedrol; Schering) administered $4.7 \%$ of the time. Eighteen millligrams of Celestone and $3 \mathrm{~mL}$ of Sensorcaine (bupivacaine HCL 0.25\%; APP Pharmaceuticals, Schaumburg, Illinois) 


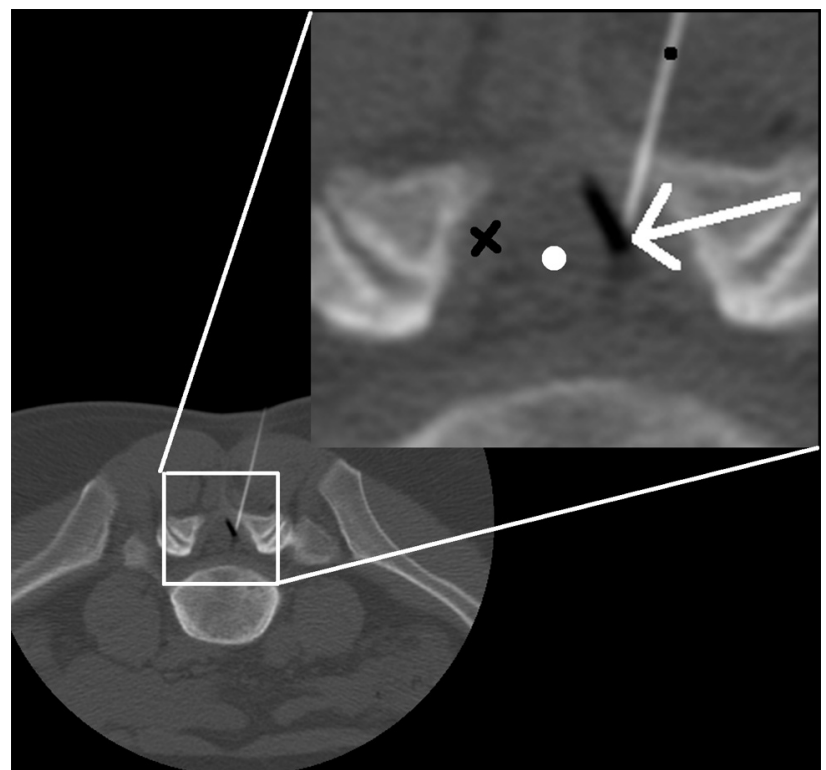

Fig 1. Axial CT image through $\mathrm{L} 5 / \mathrm{S} 1$ (3-mm section, $100 \mathrm{KVp}, 50 \mathrm{~mA})$. Image demonstrates 22-g spinal needle (black dot) traversing the ligamentum flavum (black X) tip in the epidural space. The white arrow demonstrates the interface of injected air (black) on top of epidural fat (gray). The air in the epidural space pushes the thecal sac medially (white dot).

were given in most cases $(65.2 \%)$. Of the 1000 procedures identified, no immediate or delayed clinically significant complications were reported during a standard 24-hour and 1-week follow-up (99\% of patients had 24-hour follow-up and 93\% had 2-week follow-up via phone or office consultation).

Procedure time averaged 15 minutes (range, 10-32 minutes). Average radiation dose measured as an effective dose calculated from the dose-length product and abdomen and pelvis conversion was $1.5 \mathrm{mSv}$ (range, $0.9-3 \mathrm{mSv}$ ). ${ }^{5}$

\section{Discussion}

Air localization is a technique used in conjunction with CT guidance to identify the epidural space and verify correct needle placement. This technique provides an alternative to iodine-based contrasts.

In $87 \%$ of cases, the epidural space was visualized by using air alone. The remainder required contrast to verify needle localization to the epidural space. When we compared the groups with air and contrast, the distribution of ages and levels treated was the same. Further demographic analysis did not demonstrate a clear difference between the groups. More severe spondylosis was suspected as a cause for increased contrast use; this information was not collected. Further analysis and prospective evaluation may be required to determine in which patients contrast should be used as the primary agent for localizing the epidural space.

In general, ESIs have a very low rate of complications, though they are not completely free from risk. Reported complications include spinal headache after dural puncture, infection, intravascular injections, and nerve trauma. ${ }^{6}$ There have also been reports of rarer but usually more serious complications, including pneumocephalus, ${ }^{7}$ epidural hematomas, ${ }^{8}$ paraplegia, ${ }^{9}$ vision loss, ${ }^{10}$ epidural abscess, ${ }^{11}$ and meningitis. ${ }^{12}$

While the relative risks and complications of ESI have been described in the literature, the safety of the air localization technique for use in ESI has not been previously discussed, to our knowledge. Our study detected no clinically significant complications with the use of air localization or with the procedure overall. Other studies have reported overall complication rates from $0.04 \% .^{13}$

The reported incidence of complications is very low, and this study reproduced that finding. In theory, air should reduce the risk of anaphylactic reactions to the contrast medium, and it may also reduce the rate of other adverse drug reactions from contrast. However, given the retrospective nature of this study, it is uncertain whether the lower rate of complications is attributable to the use of air in place of contrast or whether some other factor is influencing the results.

Several studies have evaluated the safety of contrast. Currently, nonionic contrast is preferred over ionic contrast due to a lower rate of ADRs. ${ }^{14}$ A large-scale study of 337,647 cases of intravenous contrast use found the ionic contrast ADR rate to be $12.66 \%$ and the nonionic $\mathrm{ADR}$ rate to be $3.13 \%$; severe ADRs were found in $0.22 \%$ of the ionic and $0.04 \%$ of the nonionic groups. ${ }^{15}$ The overall incidence of ADRs from contrast is low, though ADRs were found to be more likely in patients with a history of ADRs to contrast and in those with a history of allergy. ${ }^{15}$ One advantage of air localization is that it reduces the potential of anaphylactic reactions due to contrast allergies, and this may play a role in the reduced rates of complications we observed.

Although contrast such as gadolinium is available for use in patients with contrast allergies, ${ }^{15}$ gadolinium has the disadvantage of being significantly more expensive than regular contrast. Even a commonly used nonionic contrast such as Omnipaque- 180 costs $\$ 431$ for ten $10 \mathrm{~mL}$ vials, and the cumulative cost can be significant for an institution that performs a large volume of ESIs. Another benefit of air as a localizing agent is its cost-saving potential.

The use of CT guidance for ESIs offers many potential advantages. The ability to use air as a contrast agent is one. Another is precise needle guidance to actual soft-tissue structures, instead of inference from bony landmarks or contrast location. Room time was similar to that of a CT scanner in the hospital radiology department. Radiation dose demonstrated in this large series was reasonable compared with that found by Kim et al $^{16}$ and should not be a deterrent to CT use. Our results of $1.5 \mathrm{mSv}$ are similar to the typical effective dose from continuous fluoroscopic guidance for ESI reported by Schmid et al (0.43-1.25 mSv for $1-3$ minutes of guidance). ${ }^{17}$

\section{Conclusions}

The use of air to localize the epidural space in CT-guided ESIs has a high success rate and a very low rate of complications.

\section{References}

1. Koes BW, Scholten RJ, Mens JM, et al. Efficacy of epidural steroid injections for low-back pain and sciatica: a systematic review of randomized clinical trials. Pain 1995;63:279-88

2. Abram S. Treatment of lumbosacral radiculopathy with epidural steroids. Anesthesiology 1999;91:1937-41

3. White AH, Derby R, Wynne G. Epidural injections for the diagnosis and treatment of low back pain. Spine 1980;5:67-86

4. Furman M, O'Brien E, Zgleszewski T. Incidence of intravascular penetration in transforaminal lumbosacral epidural steroid injections. Spine (Phila Pa 1976) 2000;25:2628-32

5. Mettler FA Jr, Huda W, Yoshizumi TT, et al. Effective doses in radiology and diagnostic nuclear medicine: a catalog. Radiology 2008;248:254-63

6. Goodman BS, Posecion LW. Mallempati S, et al. Complications and pitfalls of 
lumbar interlaminar and transforaminal epidural injections. Curr Rev Musculoskelet Med 2008;1:212-22. Epub 2008 Aug 15

7. Simopoulos T, Peeters-Asdourian C. Pneumocephalus after cervical epidural steroid injection. Anesth Analg 2001;92:1576-77

8. Williams K, Jackowski A, Evans P. Epidural haematoma requiring surgical decompression following repeated cervical epidural steroid injections for chronic pain. Pain 1990;42:197-99

9. Tripathi M, Nath S, Gupta R. Paraplegia after intracord injection during attempted epidural steroid injection in an awake-patient. Anesth Analg 2005; 101:1209-11, table of contents

10. Young W. Transient blindness after lumbar epidural steroid injection: a case report and literature review. Spine (Phila Pa 1976) 2002;27:E476-77

11. Hooten W, Kinney M, Huntoon M. Epidural abscess and meningitis after epidural corticosteroid injection. Mayo Clin Proc 2004;79:682-86

12. White A, Derby R, Wynne G. Epidural injections for the diagnosis and treatment of low-back pain. Spine (Phila Pa 1976);5:78-86
13. el-Khoury G, Ehara S, Weinstein J, et al. Epidural steroid injection: a procedure ideally performed with fluoroscopic control. Radiology 1988;168: 554-57

14. Wolf G, Mishkin M, Roux S, et al. Comparison of the rates of adverse drug reactions: ionic contrast agents, ionic agents combined with steroids, and nonionic agents. Invest Radiol 1991;26:404-10

15. Safriel Y, Ali M, Hayt M. Gadolinium use in spine procedures for patients with allergy to iodinated contrast: experience of 127 procedures. AJNR Am J Neuroradiol 2006;27:1194-97

16. Kim S, Toncheva G, Anderson-Evans C, et al. Kerma area product method for effective dose estimation during lumbar epidural steroid injection procedures: phantom study. AJR Am J Roentgenol 2009;192:1726-30

17. Schmid G, Schmitz A, Borchardt D, et al. Effective dose of CT- and fluoroscopyguided perineural/epidural injections of the lumbar spine: a comparative study. Cardiovasc Intervent Radiol 2006:29:84-91 